\title{
THE USE OF REGRESSION METHODS TO STUDY GENOTYPE-ENVIRONMENT INTERACTIONS
}

\author{
EXTENDING GRIFFING'S MODEL FOR DIALLEL CROSS \\ EXPERIMENTS AND TESTING AN EMPIRICAL GROUPING METHOD
}

\author{
C. S. LIN, M. R. BINNS, and B. K. THOMPSON \\ Statistical Research Service, Research Branch Agriculture Canada, \\ Ottawa, Ontario K1A 0C5
}

Received 16.ix.76

\begin{abstract}
Summary
A model combining features of Griffing's diallel cross analysis with regression analysis for genotype-environment interactions is introduced using carp data of Moav et al. (1975) as an example. An analysis of variance based on this model provides information on the combining abilities of genetic effects and the interactions of these effects with environments from which inferences can readily be made on heterosis and heterosis-environment interactions.

Applying the empirical grouping method of Lin and Thompson (1975) to these data (ignoring their diallel cross structure) established groups which were remarkably consistent with their members' crossing backgrounds.
\end{abstract}

\section{INTRODUCTION}

REGRESSION techniques for studying genotype-environment (GE) interactions are among the most widely used methods for investigating the response patterns of genotypes. Typically, the linear regression of the data on environmental indices is obtained for each genotype (the indices are usually defined as the differences between the environmental means and the grand mean). The regression slopes and intercepts, the latter simply the genotype means, are considered to be characteristics of the genotypes.

Several recent publications have used this approach on data from diallel cross experiments. These studies generally treated the intercept and slope obtained from the regression analysis as a starting point and then proceeded with a genetic analysis treating the two estimates as if they were independent observations. For example, Hinkelmann (1974) suggested an analysis in which the sum of squares for heterogeneity of slopes was partitioned into general and specific combining ability (g.c.a. and s.c.a.) components to investigate whether heterogeneity of slope can be attributed to "interactions between environments and additive or non-additive gene action ", Moav, Hulata, and Wohlfarth (1975) regarded each slope as being the sum of two components (one proportional to the corresponding intercept and the other equal to the remainder) which they obtained by regressing the slopes on the intercepts; the two components of the slopes, and the intercepts, were then analysed separately using potence ratios to investigate the heterosis of crossbreds. Connolly and Jinks (1975) partitioned the slope into the same two components, but used the approach of Jinks (1954) and Hayman (1954) to the diallel cross to interpret them.

In view of these studies, it seems desirable to develop a single model which specifically relates the regression parameters of the GE interaction 
to the appropriate genetic parameters so that the genetic components and their interactions with environment can be examined simultaneously. One such model based on the concept of additivity and dominance was proposed by Perkins (1970), as an extension of Perkins and Jinks (1968). Here we present another model of this type based on Griffing's (1956) combining ability approach, and illustrate its practical value using the carp weight gain data of Moav et al. The relationship between this model and other models pertaining to GE interaction are discussed.

Lin and Thompson (1975), working in a more general context, have shown how the genotype-environment interactions can be used empirically to group genotypes with similar response patterns. Since this diallel cross data of Moav et al. provide a useful example of GE interactions with known genotypic background, the method of Lin-Thompson has been applied to these data to determine if the resulting groups conform with this background.

\section{Data and analyses}

Data given by Moav et al. (1975) for the adjusted weight gain of common carp (Cyprinus carpio L.) were used in the present study. Twelve genotypes, including four parental lines-a Chinese strain, Big-Belly (BB), and the European strains, Našice (Nas), Gold (G) and Dor 70 (Dor), six $F_{1}$ crossbreds

TABLE 1

Weight gain data of 12 genotypes grown under five environments (in grams, adjusted for initial weight) from Moav et al.

(1975). The final two columns are estimates of individual regressions on the environmental indices

\begin{tabular}{lc}
\multicolumn{2}{c}{ Genotype } \\
Cross & $\begin{array}{c}i j- \\
\text { identification }\end{array}$ \\
$\mathrm{BB}$ & 11 \\
$\mathrm{BB} \times \mathrm{Nas}$ & 12 \\
$\mathrm{BB} \times \mathrm{G}$ & 13 \\
$\mathrm{Nas}$ & 14 \\
$\mathrm{Nas} \times \mathrm{G}$ & 22 \\
Nas $\times$ Dor & 23 \\
$\mathrm{G} \times$ Dor & 24 \\
$\mathrm{G} \times$ Dor & 33 \\
V & 34 \\
L $($ G $\times$ Blue-Grey) & 44 \\
Environmental & \\
indices $\left(\theta_{k}\right)$ &
\end{tabular}

\begin{tabular}{|c|c|c|c|c|c|c|}
\hline \multicolumn{5}{|c|}{ Environment } & \multicolumn{2}{|c|}{ Regression } \\
\hline $\begin{array}{c}\text { Density } 1 \\
\text { at Dor }\end{array}$ & Yehiam & $\begin{array}{c}\text { Density } 2 \\
\text { at Dor }\end{array}$ & $\begin{array}{c}\text { Density } 3 \\
\text { at Dor }\end{array}$ & $\begin{array}{c}\text { Density } 4 \\
\text { at Dor }\end{array}$ & $\begin{array}{c}\text { Intercept } \\
\text { (mean) }\end{array}$ & Slope \\
\hline $\begin{array}{l}264 \\
378 \\
321 \\
383 \\
279 \\
397 \\
399 \\
301 \\
353 \\
394 \\
356 \\
360\end{array}$ & $\begin{array}{l}283 \\
395 \\
405 \\
386 \\
292 \\
397 \\
403 \\
317 \\
388 \\
403 \\
416 \\
397\end{array}$ & $\begin{array}{l}297 \\
454 \\
401 \\
457 \\
352 \\
520 \\
477 \\
391 \\
472 \\
517 \\
456 \\
444\end{array}$ & $\begin{array}{l}367 \\
505 \\
495 \\
535 \\
479 \\
590 \\
594 \\
478 \\
587 \\
593 \\
589 \\
551\end{array}$ & $\begin{array}{l}468 \\
725 \\
726 \\
740 \\
795 \\
891 \\
918 \\
726 \\
780 \\
874 \\
877 \\
816\end{array}$ & $\begin{array}{l}335 \cdot 8 \\
491 \cdot 4 \\
469 \cdot 6 \\
500 \cdot 2 \\
439 \cdot 4 \\
559 \cdot 0 \\
558 \cdot 2 \\
442 \cdot 6 \\
516.0 \\
556.2 \\
538.8 \\
513.6\end{array}$ & $\begin{array}{l}0.4773 \\
0.8030 \\
0.8855 \\
0.8490 \\
1.2285 \\
1.1639 \\
1.2406 \\
0.9962 \\
0.9858 \\
1.1238 \\
1.1910 \\
1.0555\end{array}$ \\
\hline$-144 \cdot 65$ & $-119 \cdot 90$ & -56.90 & $36 \cdot 85$ & $284 \cdot 60$ & & \\
\hline
\end{tabular}

of these lines, and two commercial crossbreds $\mathrm{V}$ and $\mathrm{L}$-were reared under five environmental conditions: two locations, Dor and Yehiam, with four population densities at Dor. The genotype-environment means are presented in table 1 . The environmental indices $\left(\theta_{k}\right)$ were obtained by subtracting the overall mean from each environmental mean. These values will be regarded as constant and assumed to represent environments in both Analyses I and II. In Analysis I, this is a slight departure from common practice where indices are usually based on the genotypes included in the analysis. However, in order to simplify the presentation of results and to facilitate comparison of the results with those of Moav et al., a single set of $\theta_{k}$ has been used throughout. Analysis I was, in fact, repeated using indices based on the 10 relevant genotypes, but the results differed little from those presented here. 
(i) Analysis I. Joint combining ability and regression analysis

The 10 genotypes of Moav et al. which comprised a diallel cross (i.e. excluding $V$ and $L$ ) were investigated in Analysis I. Let $Y_{i j k}$ be the weight gain of the $i j$ th genotype (the progeny of the cross between the $i$ th and the $j$ th parental lines) at the $k$ th environment. If the GE interaction can be expressed as a linear function of the environmental indices, the two-way classification model can be written as follows:

$$
Y_{i j k}=\mu+\alpha_{i j}+\varepsilon_{k}+\beta_{i j} \theta_{k}+\Delta_{i j k}
$$

where $\alpha_{i j}$ are the genetic effects, $\varepsilon_{k}$ are the environmental effects, $\beta_{i j}$ are the coefficients of regression of $\left(Y_{i j k}-\bar{Y}_{i j .}-\bar{Y}_{. . k}+\bar{Y}_{. . .}\right)$on $\theta_{k}$ and $\Delta_{i j k}$ are deviations.

If genetic effects are written in terms of g.c.a. and s.c.a., i.e.

$$
\alpha_{i j}=g_{i}+g_{j}+s_{i j},
$$

where $g_{i}$ and $s_{i j}$ represent g.c.a. and s.c.a. respectively; and if the interaction between these components and environment can be expressed as a linear function of $\theta_{k}$, Model 1 can be written as follows:

$$
Y_{i j k}=\mu+g_{i}+g_{j}+s_{i j}+\varepsilon_{k}+\left[\beta_{(g) i}+\beta_{(g) j}+\beta_{(s) i j}\right] \theta_{k}+\Delta_{i j k}
$$

where $\beta_{(g) i}, \beta_{(s) i j}$ are the regression coefficients of $g_{i}$ and $s_{i j}$ on $\theta_{k}$ respectively. To be consistent with Griffing's Method 2* (1956), the following restrictions are imposed:

and

$$
\sum_{i} g_{i}=\sum_{i} \beta_{(g) i}=\sum_{k} \varepsilon_{k}=0
$$

where

$$
s_{i i}+\sum_{j} s_{i j}=0, \quad \beta_{(s) i i}+\sum_{j} \beta_{(s) i j}=0
$$

$$
s_{i j}=s_{j i}, \quad \beta_{(s) i j}=\beta_{(s) j i}
$$

The analysis of variance of the carp data, assuming Model 2, is shown in table 2. Although the same mean squares would be obtained by Hinkelmann's approach, his specification of the model differs from Model 2, a point which will be discussed later. The estimates of $g_{i}$ and $s_{i j}$ at each environment and their coefficients of regression on $\theta_{k}$ are shown in table 3 . Both the g.c.a. and s.c.a. mean squares are highly significant $(P<0.01)$ when tested against the pooled residual mean square (345.4 with 27 d.f.). Most of the $g_{i} \times E$ interaction is accounted for by heterogeneity of the slopes of $g_{i}$. On the other hand, since the mean square for differences among $\beta_{(s) i j}$ (viz. 693.4) is not significant, the null hypothesis $\beta_{(s) i j}=0$, for all $i$ and $j$, is not unreasonable. It is worth noting that the pooled residual mean square is about the same magnitude as the mean square for the replication-genotype interaction pooled over environments at Dor as reported in the original paper $(1563 / 4=390 \cdot 8)$.

* Although Analysis I was based on Griffing's Method 2, Model 2 can clearly be used with any of Griffing's other three methods.

$38 / 3-\mathrm{c}$ 
TABLE 2

ANOVA for Model 2 of text

$\begin{array}{lcccc}\quad \text { Source } & & \text { d.f. } & \text { MS } \\ \text { Environment }(E) & 4 & & & 287558 \cdot 8 \\ \text { Genotypes }(G) & 9 & & & \\ \text { g.c.a. }\left(g_{i}\right) & & 3 & & 43749 \cdot 5 \\ \text { s.c.a. }\left(s_{i j}\right) & & 6 & & 14356 \cdot 7 \\ G \times E & 36 & & & \\ g_{i} \times E & & 12 & & (4901 \cdot 4) \\ \quad \text { Slopes of } g_{i} & & & 3 & 18535 \cdot 9 \\ \quad \text { Residual } & & & 9 & 356 \cdot 6 \\ s_{i j} \times E & & 24 & & (428 \cdot 2) \\ \quad \text { Slopes of } s_{i j} & & & 6 & 693 \cdot 4 \\ \quad \text { Residual } & & & 18 & 339 \cdot 9 \\ \text { Total } & & & & \end{array}$

TABLE 3

Estimates of combining abilities $\left(\mathrm{g}_{\mathbf{i}}, \mathrm{s}_{\mathbf{i j}}\right)$ and heterosis $\left(\mathrm{H}_{\mathrm{ij}}\right)$ at each environment and their coefficients of regression on the environmental indices

\begin{tabular}{|c|c|c|c|c|c|c|c|}
\hline & $\begin{array}{l}\text { Density l } \\
\text { at Dor }\end{array}$ & Yehiam & $\begin{array}{c}\text { Density } 2 \\
\text { at Dor }\end{array}$ & $\begin{array}{l}\text { Density } 3 \\
\text { at Dor }\end{array}$ & $\begin{array}{l}\text { Density } 4 \\
\text { at Dor }\end{array}$ & Mean & $\begin{array}{l}\text { Regression } \\
\text { coefficients }\end{array}$ \\
\hline $\begin{array}{l}g_{1} \\
g_{2}\end{array}$ & $-20 \cdot 8$ & $-13 \cdot 8$ & $-43 \cdot 8$ & $-57 \cdot 1$ & $-115 \cdot 8$ & $-50 \cdot 2$ & -0.2308 \\
\hline $\begin{array}{l}g_{2} \\
g_{3}\end{array}$ & $\begin{array}{r}-0 \cdot 4 \\
-10 \cdot 3\end{array}$ & $\begin{array}{l}-9.3 \\
-1.8\end{array}$ & $\begin{array}{c}-2 \cdot 3 \\
1 \cdot 0\end{array}$ & $\begin{array}{l}5 \cdot 9 \\
2 \cdot 8\end{array}$ & $\begin{array}{r}50 \cdot 4 \\
4 \cdot 6\end{array}$ & $\begin{array}{r}8.9 \\
-0.7\end{array}$ & $\begin{array}{l}0 \cdot 1313 \\
0.0252\end{array}$ \\
\hline$g_{4}$ & $\begin{array}{r}-10 \cdot 3 \\
31 \cdot 4\end{array}$ & 24.8 & $45 \cdot 2$ & $48 \cdot 4$ & $60 \cdot 8$ & $42 \cdot 1$ & 0.0744 \\
\hline$s_{11}$ & $-41 \cdot 4$ & $-56 \cdot 4$ & $-49 \cdot 1$ & $-41 \cdot 1$ & $-64 \cdot 8$ & $-50 \cdot 6$ & -0.0365 \\
\hline$s_{12}$ & $52 \cdot 3$ & $51 \cdot 1$ & $66 \cdot 4$ & 33.9 & $26 \cdot 0$ & $45 \cdot 9$ & -0.0728 \\
\hline$s_{13}$ & $5 \cdot 1$ & $53 \cdot 6$ & $10 \cdot 0$ & $27 \cdot 0$ & $72 \cdot 9$ & $33 \cdot 7$ & $0 \cdot 1158$ \\
\hline$s_{14}$ & $25 \cdot 4$ & $8 \cdot 1$ & $21 \cdot 9$ & $21 \cdot 4$ & $30 \cdot 7$ & $21 \cdot 5$ & $0 \cdot 0300$ \\
\hline$s_{22}$ & $-67 \cdot 1$ & $-56 \cdot 4$ & $-77 \cdot 1$ & $-55 \cdot 1$ & $-70 \cdot 1$ & $-65 \cdot 2$ & -0.0094 \\
\hline$s_{23}$ & $60 \cdot 8$ & $41 \cdot 1$ & $87 \cdot 5$ & $59 \cdot 0$ & $71 \cdot 7$ & $64 \cdot 0$ & $0 \cdot 0321$ \\
\hline$s_{24}$ & $21 \cdot 1$ & $20 \cdot 6$ & $0 \cdot 4$ & $17 \cdot 4$ & $42 \cdot 5$ & $20 \cdot 4$ & $0 \cdot 0596$ \\
\hline$s_{33}$ & $-25 \cdot 4$ & $-40 \cdot 4$ & $-44 \cdot 8$ & $-49 \cdot 8$ & $-47 \cdot 5$ & $-42 \cdot 8$ & -0.0294 \\
\hline$s_{34}$ & $-15 \cdot 1$ & -1.9 & $-8 \cdot 0$ & $13 \cdot 5$ & $-49 \cdot 6$ & $-12 \cdot 2$ & -0.0891 \\
\hline$s_{44}$ & $-15 \cdot 7$ & $-13 \cdot 4$ & $-7 \cdot 1$ & $-26 \cdot 1$ & $-11 \cdot 8$ & $-14 \cdot 8$ & -0.0003 \\
\hline$H_{12}$ & $106 \cdot 5$ & $107 \cdot 5$ & $129 \cdot 5$ & $82 \cdot 0$ & $93 \cdot 5$ & $103 \cdot 8$ & -0.0499 \\
\hline$H_{13}$ & $38 \cdot 5$ & $105 \cdot 0$ & $57 \cdot 0$ & $72 \cdot 5$ & $129 \cdot 0$ & $80 \cdot 4$ & $0 \cdot 1488$ \\
\hline$H_{14}$ & $54 \cdot 0$ & $43 \cdot 0$ & $50 \cdot 0$ & $55 \cdot 0$ & $69 \cdot 0$ & $54 \cdot 2$ & 0.0484 \\
\hline $\mathrm{H}_{23}$ & $107 \cdot 0$ & $92 \cdot 5$ & $148 \cdot 5$ & $111 \cdot 5$ & $130 \cdot 5$ & $118 \cdot 0$ & 0.0515 \\
\hline$H_{24}$ & $62 \cdot 5$ & $55 \cdot 5$ & $42 \cdot 5$ & $58 \cdot 0$ & $83 \cdot 5$ & $60 \cdot 4$ & 0.0644 \\
\hline$H_{34}$ & $5 \cdot 0$ & $28 \cdot 0$ & $18 \cdot 0$ & $51 \cdot 5$ & $-20 \cdot 0$ & $16 \cdot 6$ & -0.0742 \\
\hline$\theta_{k}$ & -144.6 & $-119 \cdot 9$ & $-56 \cdot 9$ & $36 \cdot 8$ & $284 \cdot 6$ & 0 & \\
\hline
\end{tabular}

(ii) Analysis II. Phenotypic grouping of genotypes based on Lin-Thompson's method

All 12 genotypes were included in this analysis. The intercepts and slopes of the regression of $Y_{i j k}$ on $\theta_{k}$ for each genotype are shown in table 1; the dendrogram of the cluster analysis is given in fig. 1. Six groups were formed when the clustering was stopped after the dissimilarity index exceeded the critical ( 5 per cent) F-value (table 4 ). This grouping, established by numerical means, seems genetically very reasonable: the four parental lines all belong to different groups; three crossbreds between Chinese and 
DISSIMILARITY INDEX $(\%)$

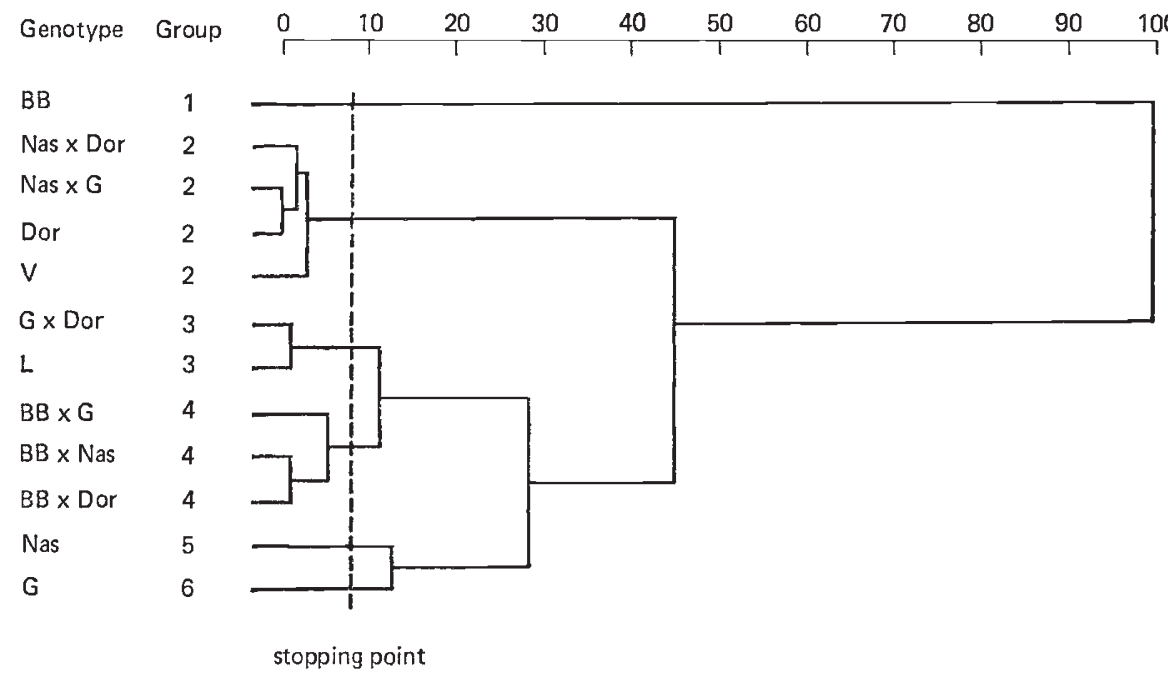

FIG. 1.-Dendrogram of clustering process for 12 genotypes, based on the Lin-Thompson method.

European strains are in one group; and European crossbreds fall into two groups. Within each of groups 2, 3, and 4, almost all of the members are half-sibs.

TABLE 4

Intercepts and slopes for the six groups of 12 genotypes

\begin{tabular}{clcc} 
Group & \multicolumn{1}{c}{ Genotype } & Intercept & Slope \\
1 & BB & $335 \cdot 8$ & 0.4773 \\
2 & Nas $\times$ G, Nas $\times$ Dor, Dor, V & $553 \cdot 1$ & $1 \cdot 1798$ \\
3 & G $\times$ Dor, L (G $\times$ Blue-Grey) & 514.8 & $1 \cdot 0207$ \\
4 & BB $\times$ Nas, BB $\times$ G, BB $\times$ Dor & $487 \cdot 1$ & $0 \cdot 8458$ \\
5 & Nas & $439 \cdot 4$ & $1 \cdot 2285$ \\
6 & G & 442.6 & 0.9965
\end{tabular}

\section{Discussion}

(i) Examination of heterosis and heterosis by environment interactions

When the use of Model 2 is justified, the approach followed in Analysis I can assist considerably in the interpretation of the various genetic relationships suggested by the data. Consider, for example, the question of heterosis.

If we define the heterosis $H_{i j k}$ resulting from crossing the $i$ th and the $j$ th parental line at the $k$ th environment as the expectation of

$$
Y_{i j k}-\frac{1}{2}\left(Y_{i i k}+Y_{j j k}\right)
$$

then under Model 2 (indicating estimates by ${ }^{\wedge}$ ), $H$ may be estimated as

or

$$
\hat{H}_{i j k}=\hat{s}_{i j}-\frac{1}{2}\left(\hat{s}_{i i}+\hat{s}_{j j}\right)+\left[\hat{\beta}_{(s) i j}-\frac{1}{2}\left(\hat{\beta}_{(s) i i}+\hat{\beta}_{(s) j j}\right)\right] \theta_{k}
$$

$$
\hat{H}_{i j k}=\bar{H}_{i j .}+\hat{\beta}_{(H) i j} \theta_{k}
$$


where

and

$$
H_{i j .}=\hat{s}_{i j}-\frac{1}{2}\left(\hat{s}_{i i}+\hat{s}_{j j}\right)
$$

$$
\hat{\beta}_{(H) i j}=\hat{\beta}_{(s) i j}-\frac{1}{2}\left(\hat{\beta}_{(s) i i}+\hat{\beta}_{(s) j j}\right) .
$$

These relationships indicate that if heterosis is a linear function of $\theta_{k}$, the estimated intercept is a linear combination of $\hat{s}_{i j}$ and the estimated slope is a linear combination of $\hat{\beta}_{(s)} i j$.

Although the concepts and calculations presented by Perkins (1970) for the diallel cross differ considerably from those presented here, it is,interesting to note that $\bar{H}_{i j}$. is identical to Perkins' estimate of dominance, and if the same environmental indices are used in both methods, $\hat{\beta}_{(H)_{i j}}$ is identical to Perkins' estimate of the linear coefficient of dominance. These points are discussed below.

An interesting feature of Analysis I was that $g_{i}$ differed significantly among environments but $s_{i j}$ did not; i.e. the $\beta_{(s)}$ ij appeared to be zero under this range of environmental conditions (table 3 ). Hence, it is apparent from the expression for $H_{i j k}$ above that there was no heterosis by environment interaction. This implies that from the point of view of adaptation, only the g.c.a. was important, or alternatively, that heterosis remained constant over environments.

Again, since the $\beta_{(s)_{i j}}$ were not significantly different from zero, an appropriate model for $\beta_{i j}$ of Model 1 is $\beta_{i j}=\beta_{(g) i}+\beta_{(g) j}$ which implies that $\beta_{i j}=\frac{1}{2}\left(\beta_{i i}+\beta_{j j}\right)$, since $\beta_{i i}=2 \beta_{(g) i}$, if $\beta_{(s)} i i=0$. This relationship explains why Moav et al. found the response slopes of crossbreds to be generally intermediate between those of their parents.

\section{(ii) Comparison with Moav's et al. potence ratio analyses}

Moav et al. used a potence ratio to compare the weight gains of six crossbreds at six environmental points: three actual points and three hypothetical points (Moav et al., p. 334). The ratio at the $k$ th environment is defined as $D_{i j k} / A_{i j k}$, where $D_{i j k}$ is equivalent to the present $H_{i j k}$ and $A_{i j k}$ is defined as one-half of the difference between parents. They observed that the mean ratio of all six crosses became smaller as the environments improved, and that in general this held true for each individual cross.

This trend can be interpreted in terms of Model 2 as follows. Since it has already been shown that $H_{i j k}$ (and $\beta_{(s) i j}$ ) do not appear to change with respect to environments, any change in the potence ratio is due to $A_{i j k}$ which can be written,

$$
\hat{A}_{i j k}=\hat{g}_{i}-\hat{g}_{j}+\frac{1}{2}\left(\hat{s}_{i i}-\hat{s}_{j j}\right)+\left[\hat{\beta}_{(g) i}-\hat{\beta}_{(g) j}+\frac{1}{2}\left(\hat{\beta}_{(s) t i}-\hat{\beta}_{(s) j j}\right)\right] \theta_{k} .
$$

A change in $A_{i j k}$ due to environment is merely a change in $\left(\hat{\beta}_{(g) i}-\hat{\beta}_{(g) j}\right) \theta_{k}$

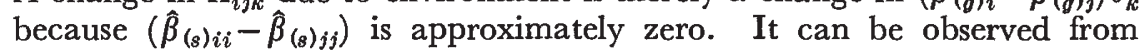
table 3 that differences among $\hat{g}_{i}$ increased as $\theta_{k}$ improved, particularly when one parent was $B B$.

Moav et al. also investigated the potence ratios of the two components of slope mentioned in the first section (the slope, $\hat{\beta}_{i j}+1$ of Model 1 , assuming $\theta_{k}=\varepsilon_{k}$, is redefined to be equal to $1+M \hat{\alpha}_{i j}+N_{i j}$ where $M$ is estimated by the least squares regression of $\hat{\beta}_{i j}$ on $\hat{Q}_{i j}$ and $N_{i j}$ is the remainder). They 
observed that for six crossbreds the potence ratios of the scale function $\left(1+M \hat{\alpha}_{i j}\right)$ were all positive, while the potence ratios of the specific responsiveness $\left(N_{i j}\right)$ were all negative. The mean potence ratios were 1.30 and -1.12 respectively. From these results, they concluded that both components expressed heterosis of about the same magnitude but in opposite directions giving rise to "additivity of slopes", that is, the absence of heterosis-environment interactions. Thus Moav et al. assume two genetic systems working independently, one controlling the intercept and part of the slope (scale function) and the other controlling the rest of the slope (specific responsiveness), while the present interpretation assumes that one genetic system (g.c.a. and s.c.a.) controls both $\alpha_{i j}$ and $\beta_{i j}$. From Moav's et al. point of view the additivity of slope arises from the balance of two systems, whereas under the present considerations, additivity is due to nonresponsiveness of s.c.a. It is difficult to determine which of these two interpretations is more in accord with genetic reality. However, it should be noted that the present approach seems easier to comprehend as it is more consistent with the concept of combining ability proposed by earlier workers.

\section{(iii) Comparison with Moav's et al. classification}

In a brief comment, Moav et al. suggested four classes for the 12 genotypes, based on the average performance. They included groups 5 and 6 of table 4 in one class and groups 2 and 3 in another class. From fig. 1, it can be seen that groups 5 and 6 are relatively similar, but groups 2 and 3 are considerably different. For comparison ANOVAs based on the four groups of Moav et al. and the six groups of fig. 1 are presented in table 5 . The analyses show that there were still large proportions of variation in

TABLE 5

ANOVAs based on the two groupings of the genotypes. A refers to the grouping obtained in the text. $B$ refers to the grouping obtained by Moav et al

\begin{tabular}{|c|c|c|c|c|}
\hline \multirow[b]{2}{*}{ Source } & \multicolumn{2}{|c|}{ A } & \multicolumn{2}{|c|}{ B } \\
\hline & d.f. & MS & d.f. & MS \\
\hline Environments $(E)$ & 4 & $362677 \cdot 0$ & 4 & $362677 \cdot 0$ \\
\hline Groups (Gr) & 5 & $45603 \cdot 2$ & 3 & $72745 \cdot 5$ \\
\hline Genotype $(\alpha) / \mathrm{Gr}$ & 6 & $645 \cdot 1$ & 8 & $1706 \cdot 3$ \\
\hline $\mathrm{Gr} \times \mathrm{E}$ & 20 & & 12 & \\
\hline slope $\times \mathrm{Gr}$ & 5 & $12741 \cdot 2$ & 3 & 18787.7 \\
\hline dev $\times$ Gr & 15 & $201 \cdot 0$ & 9 & 165.9 \\
\hline$\alpha \times E$ & 24 & & 32 & \\
\hline slope $\times \alpha / \mathrm{Gr}$ & 6 & $262 \cdot 9$ & 8 & $1115 \cdot 0$ \\
\hline $\operatorname{dev} . \times \alpha / \mathrm{Gr}$ & 18 & $414 \cdot 0$ & 24 & 373.9 \\
\hline
\end{tabular}

"genotype within groups" and in "slope $\times \propto$ within groups" for Moav's et al. classification, indicating that the average performance and slopes within certain groups are not really the same. It may be observed from table 4 that groups 5 and 6 have similar intercepts but they differ in slope, while groups 2 and 3 have similar slopes but they differ in intercept. It should be noted that the relatively clear-cut classification pattern of Analysis II could be, in part, a result of the small heterosis by environment interaction. 
(iv) The interrelationships between Model 2 and other regression models for $G E$ interactions

When a diallel cross design is used in a GE experiment, the data can be analysed using a model such as Model 2 which incorporates genetic and regression models, or they can be analysed using a regression model such as Model 1, which ignores the information provided by the genetic structure of the experiment. In a more general context Model 1 can be expressed as a simple regression of $Y_{i j k}$ on $\theta_{k}$; i.e.

$$
Y_{i j k}=a_{i j}+b_{i j} \theta_{k}+\Delta_{i j k}^{\prime}
$$

The algebraic relationships among the estimates of the corresponding parameters in each model are summarised below:

(i) The estimates under Model 1 can be expressed in terms of the estimates of Model 3 as

$$
\begin{aligned}
& \hat{\beta}_{i j}=\hat{b}_{i j}-\sum_{k}\left(\bar{Y}_{. . k}-\bar{Y}_{. . .}\right) \theta_{k} / \sum_{k} \theta_{k}^{2}=\hat{b}_{i j}-\bar{b}_{. .} \\
& \hat{\alpha}_{i j}=\hat{a}_{i j}-\bar{a}_{. .}
\end{aligned}
$$

where $\bar{b}_{. .}$and $\bar{a}_{. .}$are the means of all estimates of $b_{i j}$ and $a_{i j}$ respectively.

When $\theta_{k}=\bar{Y}_{. . k}-\bar{Y}_{\ldots}=\hat{\varepsilon}_{k}$, the above equations become

$$
\begin{aligned}
& \hat{\beta}_{i j}=\hat{b}_{i j}-1 \\
& \hat{\alpha}_{i j}=\hat{a}_{i j}-\bar{Y}_{\ldots} .
\end{aligned}
$$

Thus, for example, in Analysis $1\left(\theta_{k} \neq \hat{\varepsilon}_{k}\right)$

$$
\hat{\beta}_{11}=0.4773-0.9754=-0.4981
$$

(ii) The $\beta_{i j}$ of Model 1 and $\beta_{(g)_{i}}$ and $\beta_{(s)_{i j}}$ of Model 2 are related as follows

$$
\hat{\beta}_{i j}=\hat{\beta}_{(g) i}+\hat{\beta}_{(g) j}+\hat{\beta}_{(s) i j}
$$

e.g. from table 3 .

$$
2 \hat{\beta}_{(g) 1}+\hat{\beta}_{(s) 11}=2(-0 \cdot 2308)-0.0365=-0.4981
$$

which is $\hat{\beta}_{11}$ of Analysis 1 .

(iii) The estimates of $\beta_{(g)}, \beta_{(s)_{i j}}$ and their sums of squares (SS) given in tables 2 and 3 can be obtained directly by regressing $g_{i k}, s_{i j k}$ on $\theta_{k}$, where $g_{i k}$ and $s_{i j k}$ represent the g.c.a. and s.c.a. respectively at the $k$ th environment as shown in the left column of table 6 . However, they can also be expressed in terms of $\hat{\beta}_{i j}$ of Model 1, or in terms of $b_{i j}$ of Model 3 as shown in the right column of table 6 .

When $\hat{b}_{i j}$ are analysed by Griffing's combining ability analysis, the estimates of g.c.a. and s.c.a. for $\hat{b}_{i j}$ are equivalent to $\hat{\beta}_{(g)_{i}}$ and $\hat{\beta}_{(s)_{i j}}$; and the sums of squares for g.c.a. and s.c.a. of $\hat{b}_{i j}$ multiplied by the constant 
TABLE 6

Formulae for $\hat{\beta}_{(\mathrm{g}) \mathrm{i}}$ and $\hat{\beta}_{(\mathrm{s}) \mathrm{ij}}$ and their sums of squares ( $\mathrm{p}$ is the number of inbred lines used in the diallel cross)

In terms of $\hat{g}_{i k}$ and $\hat{s}_{i j k}$

In terms of $\hat{b}_{i j}$

$\begin{array}{lc}\hat{\beta}_{(g) i} \hat{g}_{i k} \theta_{k} / \sum_{k} \theta_{k}^{2} & \frac{1}{p+2}\left(\sum_{j} \hat{b}_{i j}+\hat{b}_{i i}-\frac{2}{p} \sum_{i \leqq j} \sum_{j} \hat{b}_{i j}\right)=\text { g.c.a. of } \hat{b}_{i j} \\ \hat{\beta}_{(s) i j} & \sum_{k} \hat{s}_{i j k} \theta_{k} / \sum_{k} \theta_{k}^{2}\end{array}$

SS (slopes of $\left.g_{i}\right) \quad(p+2) \sum_{i}\left(\sum_{k} \hat{g}_{i k} \theta_{k}\right)^{2} / \sum \theta_{k}^{2} \frac{1}{p+2}\left\{\sum_{i}\left(\sum_{j} \hat{b}_{i j}+\hat{b}_{i j}\right)^{2}-\frac{4}{p}\left(\sum_{i \leqq j} \sum_{i j} \hat{b}_{i j}\right)^{2}\right\} \sum_{k} \theta_{k}^{2}$ $=\left(\right.$ SS of g.c.a. of $\left.\hat{b}_{i j}\right) \sum_{k} \theta_{k}^{2}$

SS (slopes of $\left.s_{i j}\right) \quad \sum_{i \leqq j} \sum_{j}\left(\sum_{k} \hat{s}_{i j k} \theta_{k}\right)^{2} / \sum \theta_{k}^{2}$

$$
\begin{aligned}
\left.\sum_{i \leqq j} \sum_{j}\left(\hat{b}_{i j}-\bar{b}_{. .}\right)^{2} \sum_{k} \theta_{k}^{2}-\mathrm{SS} \text { (slope of } g_{i}\right) \\
=\left(\text { SS of s.c.a. of } \hat{b}_{i j}\right) \sum_{k} \theta_{k}^{2}
\end{aligned}
$$

value $\Sigma \theta_{k}^{2}$, are equivalent to the sums of squares for "slope of $g_{i}$ " and for "slope of $s_{i j}$ " respectively in table 2. Furthermore, the sum of these two sums of squares is

$$
\sum_{i} \sum_{j}\left(\hat{b}_{i j}-\bar{b}_{. .}\right)^{2} \sum_{k} \theta_{k}^{2}
$$

which is the sum of squares for heterogeneity of slope under Model 1 and Model 3. Thus, when data from a diallel cross are analysed according to Model 3 or Model 1, the sum of squares for heterogeneity of slope can be partitioned into g.c.a. and s.c.a. components as suggested by Hinkelmann. Algebraically, as stated earlier, the present analysis and Hinkelmann's analysis yield the same results. The difference is that by relating regression parameters to the parameters of combining ability model, Model 2 allows a direct biological interpretation.

In contrast to the above three models which are generally described in terms of phenotypic parameters, the model of Perkins is directly related to the underlying gene action, in particular, to additive and dominance effects. However, the application of Perkins' (1970) model requires the satisfaction of certain restrictive assumptions concerning the genetic background of the material (e.g. the parental lines, unlike Moav's, must be pure-breeding). In situations where the assumptions can be justified and the researcher is interested in investigating the nature of the underlying gene action and its interaction with the environment, Perkins' analysis may be more informative. It is interesting to note that where Perkins' model is appropriate the parameter estimates are algebraically related to those of Model 2. In the 
particular form presented by Perkins, the following relationships hold (using Perkins' notation to the left of the equality sign):

$$
\begin{aligned}
& \text { additive: } \quad \hat{d}_{i}=2 \hat{g}_{i}+\hat{s}_{i i}-\sum_{i=1}^{p} \hat{s}_{i i} / p \\
& \text { dominance: } \hat{h}_{(i j)}^{\prime}=\hat{s}_{i j}-\frac{1}{2}\left(\hat{s}_{i i}+\hat{s}_{j j}\right)=\bar{H}_{i j} .
\end{aligned}
$$

and if $\theta_{k}=\varepsilon_{k}^{\prime}$, (where $\varepsilon_{k}^{\prime}$ are the environmental indices used by Perkins and are based on parental lines only)

$$
\begin{aligned}
& \text { additive } \times \text { Env.: } \quad \hat{\beta}_{d}^{\prime}=2 \hat{\beta}_{(g) i}+\hat{\beta}_{(s) i i}-\sum_{i=1}^{p} \beta_{(s) i i} / p \\
& \text { dominance } \times \text { Env.: } \hat{\beta}_{h(i j)}^{\prime}=\hat{\beta}_{(s) i j}-\frac{1}{2}\left(\hat{\beta}_{(s) i i}+\hat{\beta}_{(s) j j}\right) \\
& =\hat{\beta}_{(H) i j}
\end{aligned}
$$

\section{Conclusion}

The main advantage of using Model 2 for studying GE interactions in a diallel cross experiment is that both the combining abilities of genetic effects and the linear function of combining ability components by environment interaction can be studied simultaneously. Since the parameters representing the regression model are directly related to the corresponding parameters representing the combining abilities analysis, the model provides a direct and easy biological interpretation. Furthermore, from the results, both heterosis and heterosis by environment interactions can be easily assessed by examining linear combinations of $s_{i j}$ and $\left.\beta_{(s)}\right)_{i j}$ respectively.

It has been shown that the regression coefficients of g.c.a. and s.c.a. components on environmental indices are algebraically equivalent to the g.c.a. and the s.c.a. of $\hat{b}_{i j}$ (Model 3) or $\hat{\beta}_{i j}$ (Model 1). Thus, it can be said that Model 2 provides both a structural as well as a biological base for what may be termed a joint combining ability analyses for both intercept and slope.

The close agreement between groups obtained numerically using LinThompson's method and the parental association of genotypes reflects the importance of GE interactions as classification criteria in biological studies. This example suggests that the grouping method is a useful tool not only in simplifying the interpretation of complicated interactions but also in identifying underlying biological relationships among genotypes in the resulting groups.

\section{REFERENGES}

CONNOLly, V., AND Jinks, J. L. 1975. The genetical architecture of general and specific environmental sensitivity. Heredity, 35, 249-259.

GRIFFING, B. 1956. Concept of general and specific combining ability in relation to diallel crossing system. Aust. 7. Biol. Sci., 9, 463-493.

HAYMAN, B. I. 1954. The analysis of variance of diallel tables. Biometrics, 10, 233-244.

hinkelmann, к. 1974. Genotype-environment interaction: Aspect of statistical designs, analysis and interpretation. Paper presented to the 8th International Biometric Conference. Aug. 1974, Constanta, Romania.

JiNks, J. L. 1954. The analysis of continuous variation in a diallel cross of Nicotiana rustica varieties. Genetics, 39, 767-788. 
LIN, C. S., AND thompson, B. 1975. An empirical method of grouping genotypes based on a linear function of the genotype-environment interaction. Heredity, 34, 255-263.

MOAV, R., HUlata, G., AND WOHLFARTH, G. 1975. Genetic differences between the Chinese and European races of the common carp. 1. Analysis of genotype-environment interactions for growth rate. Heredity, 34, 323-340.

PERKINS, J. M. 1970. Environmental and genotype-environmental components of variability. VI. Diallel sets of crosses. Heredity, 25, 29-40.

PERKINS, J. M., AND JINks, J. L. 1968. Environmental and genotype-environmental component of variability. III. Multiple lines and crosses. Heredity, 23, 339-356. 